\title{
Multivariate Model Based on UV-Vis Spectroscopy and Regression in Partial Least Squares for Determination of Diameter and Polydispersity of Silver Nanoparticles in Colloidal Suspensions
}

\author{
J. F. B. Rodrigues $\mathbb{D}^{1},{ }^{1}$ E. P. S. Junior $\mathbb{D}^{2},{ }^{2}$ K. S. Oliveira, ${ }^{1}$ M. R. R. Wellen $\mathbb{D}^{3}{ }^{3}$ S. S. Simões $\mathbb{D},{ }^{2}$ \\ and M. V. L. Fook ${ }^{1}$ \\ ${ }^{1}$ Academic Unit of Materials Engineering, Federal University of Campina Grande, Campina Grande PB 58429-140, Brazil \\ ${ }^{2}$ Chemical Department, State University of Paraíba, Campina Grande PB 58429-000, Brazil \\ ${ }^{3}$ Materials Engineering Department, Federal University of Paraíba, João Pessoa PB 58051-900, Brazil \\ Correspondence should be addressed to J. F. B. Rodrigues; filipe.rodrigues@certbio.ufcg.edu.br
}

Received 28 December 2019; Revised 6 April 2020; Accepted 8 April 2020; Published 23 April 2020

Academic Editor: Zehra Durmus

Copyright (c) 2020 J. F. B. Rodrigues et al. This is an open access article distributed under the Creative Commons Attribution License, which permits unrestricted use, distribution, and reproduction in any medium, provided the original work is properly cited.

\begin{abstract}
In the universe of nanomaterials, silver nanoparticles (AgNPs) have attracted the attention of researchers because of their optical, catalytic, antimicrobial, fungicidal, and bactericidal properties. Recently, studies have correlated the toxicity and efficacy of antimicrobial activity with surface-volume ratio, morphology, polydispersity, ligand types, particle size, and stability of AgNPs. Soon, the need for characterization of properties such as diameter and polydispersity is clear. The methodologies conventionally used for characterization of AgNPs, although accurate, are generally expensive and laborious and can degrade the sample. Thus, the development of methodologies based on UV-Vis spectroscopy and chemometric techniques appears as an alternative for the characterization of diameter and polydispersity of the nanoparticles. For the development of the methodology in question, 50 samples were synthesized, varying the type, volume, and concentration of the reagents in order to increase the diameter and polydispersity values. All samples were analyzed by DLS and UV-Vis spectroscopy. For the construction of multivariate calibration models, the calibration and validation sets were selected using the SPXY algorithm, and their predictive capacity was evaluated based on the method figures. The model that presented the best predictive capacity was the one built with the pretreated spectra with the 1st derivative with a 15-point window and 2nd-order polynomial, providing prediction errors of $5.31 \%$ and $4.43 \%$ for diameter and polydispersity, respectively.
\end{abstract}

\section{Introduction}

Noble metal nanoparticles (NPs), specifically silver (Ag) and gold $(\mathrm{Au})$, have attracted the attention of distinct sciences such as biomedicine [1], engineering [2], and food technology [3] as well as several industrial fields such as cosmetics [4], electronic devices [5], and building construction [6]. The increasing interest is due to the single properties of nanoscale materials, i.e., cell viability [7], anticancer action [8], and silver nanoparticle applicability in scaffolds [9], membranes [10], and hydrogel dressings [11], for instance.
The size and distribution of silver NPs (AgNPs) are important characteristics for seeking their best applications, since they have direct influence on the bactericidal activity [12-15], while properties such as concentration, dispersion, and morphology have influence on toxicity $[16,17]$. Thus, the control and size evaluation of AgNPs are essential.

Several methodologies can be used to characterize particle size, such as transmission electron microscopy (TEM) [18, 19], scanning electron microscopy (SEM) [20, 21], atomic force microscopy (AFM) [22], visible ultra violet spectroscopy (UV-Vis), analytical ultracentrifugation (AUC) $[23,24]$, flow 
field fractionation (FFF) [25], X-ray diffraction (XRD) [26], and light dynamic scattering (LDS) [27]. Nevertheless, most of these methods require careful sample preparation (TEM, SEM, and AFM), whereas AFM, AUC, and FFF have the possibility of presenting artifacts as well as have high cost such as AFM [28-30].

UV-Vis spectroscopy and LDS are among the most applied techniques for nanoparticle characterization [31, 32]. LDS measures the scattered light by a laser that goes through the colloid by measuring the particle hydrodynamic size $[29,33]$. Related to UV-Vis spectroscopy, the light beam intensity going through the sample is measured. Both are selective techniques, fast, simple, and do not require laborious calibration, which make them widely appropriated for NP characterization [28]. However, some limitations are verified; using LDS for size determination of very polydisperse colloidal solutions is not adequate because the light scattering of larger nanoparticles is so intense that light from smaller particles is concealed.

UV-Vis spectroscopy has been, in most of the works, used to observe the maximum wavelength behavior of characteristic peaks. Haiss et al. [28] analyzed, theoretically and experimentally, the optical properties (diameter and concentration) of gold spherical nanoparticles (3 to $120 \mathrm{~nm}$ ) in an aqueous solution. The UV-Vis spectra were used for peak monitoring between 520 and $580 \mathrm{~nm}$ (surface plasmon peak) and to corroborate theoretical results. Paramelle et al. [34] developed a standardized univariate method based on $\mathrm{UV}-\mathrm{V}$ is spectroscopy to determine the size and concentration of monodispersed and stabilized AgNPs with sodium citrate, ranging in size from 8 to $100 \mathrm{~nm}$. These methodologies, although presenting good results, are based on restricted range of wavelengths.

UV-Vis spectroscopy is not able to distinguish the NPs' maximum absorption bands with different sizes, only the displacement of maximum absorption peak in comparison to monodisperse colloids [29]. Therefore, in view of the UV-Vis spectroscopy's low sensitivity to identify individual absorption bands of AgNPs with different sizes, the construction of multivariate models based on UV-Vis spectra appears as an indispensable tool able to model small displacements of absorption bands correlating them to NP size.

Multivariate data calibration is a chemometric field [35] which comprises the instrumental analysis of a large number of variables (i.e., radiation absorbance at various wavelengths) of several samples. This type of analysis seeks to relate the samples and instrumental variables contained in a data set to provide a mathematical model which can predict the modeling property in an unknown sample [36]. Multivariate models can model and eliminate interferences [37] and, therefore, often have superior predictive capacity to univariate models, since they use several variables instead of only one, which represents more information about the problem under investigation. In general, multivariate calibration methods represent noise reduction, interference modeling, the exploratory aspect, and the possible control of outliers [38].

In the literature, reports are found using UV-Vis spectroscopy in a multivariate way, together with chemometric methods for nanoparticle properties analyses. MorenoMartin et al. [13] used UV-Vis spectroscopy and PLS (Partial Least Square) regression for the development of a multivariate analytical method able to evaluate concentration and size of AgNPs (in the range from 0.62 to $2.5 \mathrm{mg} \cdot \mathrm{L}^{-1}$ and from $29 \pm 3$ to $79 \pm 7 \mathrm{~nm}$, respectively) based on AgNP surface plasmon resonance band (SPRB) changes when forming aggregated structures with L-Cys. A good relation between predicted values and added AgNPs was found. In another work, Rabbani et al. [39] used molecular absorption spectroscopy and PLS to determine gold nanoparticle size (AuNP) based on the relation between the kinetic behavior of citrate-coated L-cysteine (L-Cys) aggregation. However, up to know, no work has been found to determine the polydispersity of AgNPs using UV-Vis spectroscopy associated with chemometric tools. In addition, reported works need the interaction of L-cysteine that bonds NPs through carboxylate groups in sodium citrate to form agglomerates (Jing and Fang [40] and Rabbani et al. [39]) or can be only applied to monodispersed AuNPs or AgNPs stabilized with sodium citrate, restricting the method application.

Based on the above mentioned, this work presents a methodology based on UV spectroscopy and multivariate calibration methods, intended for the simultaneous determination of size and polydispersity of AgNPs in colloidal solutions in a fast, simple, and low cost way. The proposed methodology has as differential the determination of two important characterization properties, i.e., size and polydispersity of AgNPs from UV spectra and the ability for application to both monodisperse and polydisperse particles.

\section{Materials and Methods}

2.1. Reagents. Silver nitrite $\left(\mathrm{AgNO}_{3}\right.$, purity $\left.99.0 \%\right)$, sodium citrate $\left(\mathrm{Na}_{3} \mathrm{C}_{6} \mathrm{H}_{5} \mathrm{O}_{7} \cdot 2 \mathrm{H}_{2} \mathrm{O}\right.$, purity $\left.99.0 \%\right)$, and hydrogen peroxide $\left(\mathrm{H}_{2} \mathrm{O}_{2}, 35 \%\right)$ were purchased from Neon ${ }^{\circledR}$ (São Paulo, Brazil). Sodium borohydride $\left(\mathrm{NaBH}_{4}, \geq 96 \%\right.$ purity) was purchased from Sigma-Aldrich ${ }^{\circledR}$ (Missouri, USA). Ultrapure water was obtained from a Master System MS2000 GEHAKA $^{\circledR}$ (São Paulo, Brazil). All reagents were analytical grade.

2.2. AgNP Synthesis. AgNP synthesis was performed by a chemical reduction method based on Zhang et al. [41] following the adding order as reported in Table 1 (concentrations and volumes reported); silver nitrate, sodium citrate, hydrogen peroxide, and sodium borohydride were subsequently added, i.e., one after the other to $30 \mathrm{ml}$ of ultrapure water, under stirring (1150 rpm) for 3 minutes at room temperature $\left(23^{\circ} \mathrm{C}\right)$. After addition of sodium borohydride, change in the color solution was observed, i.e., from translucent to yellow and subsequently to dark blue. This color change was previously reported by Panzarasa [42] and can be attributed to the surface plasmon excitation which, according to Pacioni et al. [30], is characteristic of triangular-shaped nanoparticles (nanoplates).

Related to the $\mathrm{pH}$ of the synthesis mixture, ultrapure water $\mathrm{pH}$ was close to neutral, i.e., 7 . After the synthesis the solution was acidic, i.e., $\mathrm{pH}$ : 5.5. 
TABLE 1: Concentration and used volume during synthesis.

\begin{tabular}{lccc}
\hline Adding order & Reagents & Content $\left(\mathrm{mmol} \cdot \mathrm{L}^{-1}\right)$ & Range of volume content $(\mu \mathrm{L})$ \\
\hline $1^{\text {st }}$ & $\mathrm{AgNO}_{3}$ & 0.1 & $15-45$ \\
$2^{\text {nd }}$ & $\mathrm{Na}_{3} \mathrm{C}_{6} \mathrm{H}_{5} \mathrm{O}_{7}$ & $15-45$ & $500-2500$ \\
$3^{\text {rd }}$ & $\mathrm{H}_{2} \mathrm{O}_{2}$ & $35 \%$ or $1153 \mathrm{mmol} \cdot \mathrm{L}^{-1}$ & $20-150$ \\
$4^{\text {th }}$ & $\mathrm{NaBH}_{4}$ & $50-120$ & $150-300$ \\
\hline
\end{tabular}

2.3. Characterization of Silver NPs by Reference Methods. Dynamic light scattering: size and size dispersion of NPs were measured using Brookhaven's ZetaPals. The applied parameters were $25^{\circ} \mathrm{C}$ with fixed scattering angle of $90^{\circ}$, laser wavelength of $632.8 \mathrm{~nm}(\mathrm{He}-\mathrm{Ne})$, mean viscosity of $0.8887 \mathrm{mPa} \cdot \mathrm{s}$, and average refractive index of 1.330 . Analyses were performed in a quartz cuvette, and three measurements were taken to use the data average.

UV spectroscopy: spectra in the ultraviolet region were recorded in a PerkinElmer UV-Vis Lambda 35 spectrometer, in the wavelength range from 200 to $700 \mathrm{~nm}$. All analyses were performed in triplicate. The mean spectra were used to build the multivariate models, aiming at greater reproducibility and accuracy of results.

2.4. Development of Multivariate Models. Construction of multivariate models was based on the following steps: (a) selection of working range, (b) spectral preprocessing, (c) detection of anomalous samples, (d) selection of calibration set and external prediction, (e) construction of PLS models, (f) internal validation of PLS model, and (g) external validation of PLS model. Steps (a), (b), (c), (e), and (f) were performed with the software The Unscrambler ${ }^{\circledR}$ v9.1 of CAMO Process and step (d) with the aid of SPXY algorithm written in $\mathrm{MATLAB}^{\circledR}$, version 6.5 .

\section{Results and Discussion}

3.1. Samples and Reference Values. Multivariate calibration methods are aimed at developing, from instrumental data, reliable and strong models which accurately predict properties or sample characteristics. To do so, it is necessary that samples from the calibration set have an adequate variability of the dynamic parameter range under investigation. In this work, 50 samples were synthesized, with diameter ranging from $26.38 \mathrm{~nm}$ to $71.19 \mathrm{~nm}$ and polydispersity ranging from 0.021 to 0.472 . Their diameter and polydispersity were determined by conventional methods, i.e., UV-Vis and DLS spectroscopy, as described in Section 2.3.

UV-Vis spectroscopy is a widely used technique for particle characterization, especially for colloidal suspensions, and it is conventionally associated with Mie's theory $[43,44]$ for size and shape verification of AgNPs. According to Mie's theory, anisotropic particles can exhibit two or three absorption bands, according to their shape [43, 44]. Figure 1 shows the characteristic UV-Vis spectrum (300 to $1100 \mathrm{~nm}$ ) for the AgNP colloidal solution. A maximum absorption band at $742 \mathrm{~nm}$ can be observed, characteristic of almost perfect triangular silver nanoparticles [44]. Similar results were obtained by Chen and Carroll [45]. The second absorption

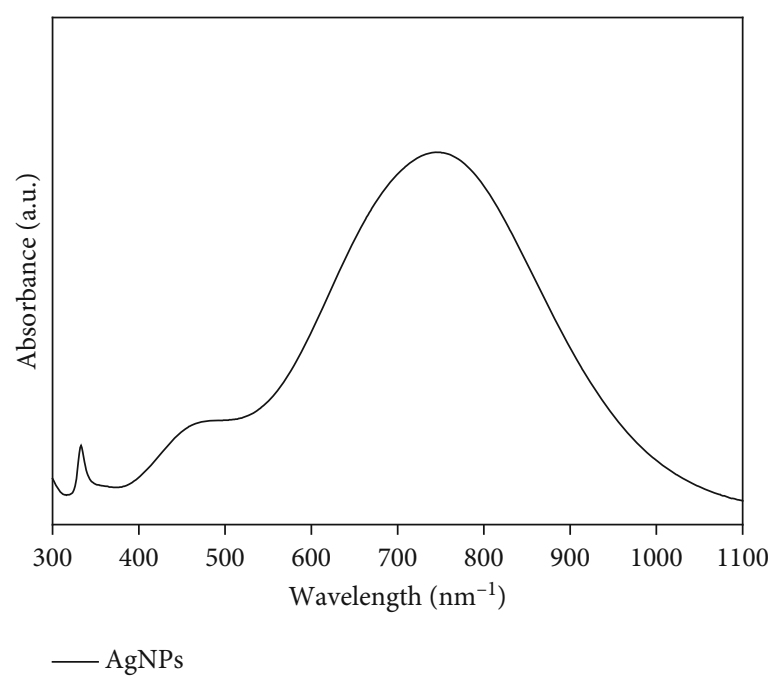

Figure 1: UV-Vis spectrum of a colloidal solution of AgNPs.

band is in the shoulder form with absorption at $465 \mathrm{~nm}$ which indicates dipole resonances, characteristic of triangular nanoparticles, according to the calculation of Chalmers and Griffiths and Farooq et al. [46, 47]. The third absorption band is located at $333 \mathrm{~nm}$ and may be associated with quadrupole resonance outside the plane [44, 45, 47].

Related to DLS results, an average diameter of $33.40 \mathrm{~nm}$ and average polydispersity of 0.179 can be observed, referring to the sample size variability in relation to its distribution by intensity. The values of polydispersity range from 0 to 1 ; the lower this value, the more monodispersed and, consequently, the less heterogeneous will be the solution [24]. Figure 2 shows the sample variability in relation to (a) diameter and (b) polydispersity.

According to the graph of Figure 2(a), all samples are within the nanoscale values, i.e., 1 to $100 \mathrm{~nm}$, most of them with diameters lower than $55 \mathrm{~nm}$. In relation to polydispersity values (Figure 2(b)), it can be noticed that the majority $(80 \%)$ of samples are in monodisperse form and the minority has polydispersity (20\%). Particles with polydispersity values lower than 0.3 can be considered monodisperse and represent particles of the same size and shape, whereas particles with values higher than 0.3 indicate solution polydispersity [48]. Sample 14 was excluded from the model because it presented values of diameter and polydispersity that differed from the average.

3.2. Construction of Multivariate Calibration Models. The construction of multivariate models based on spectroscopic 


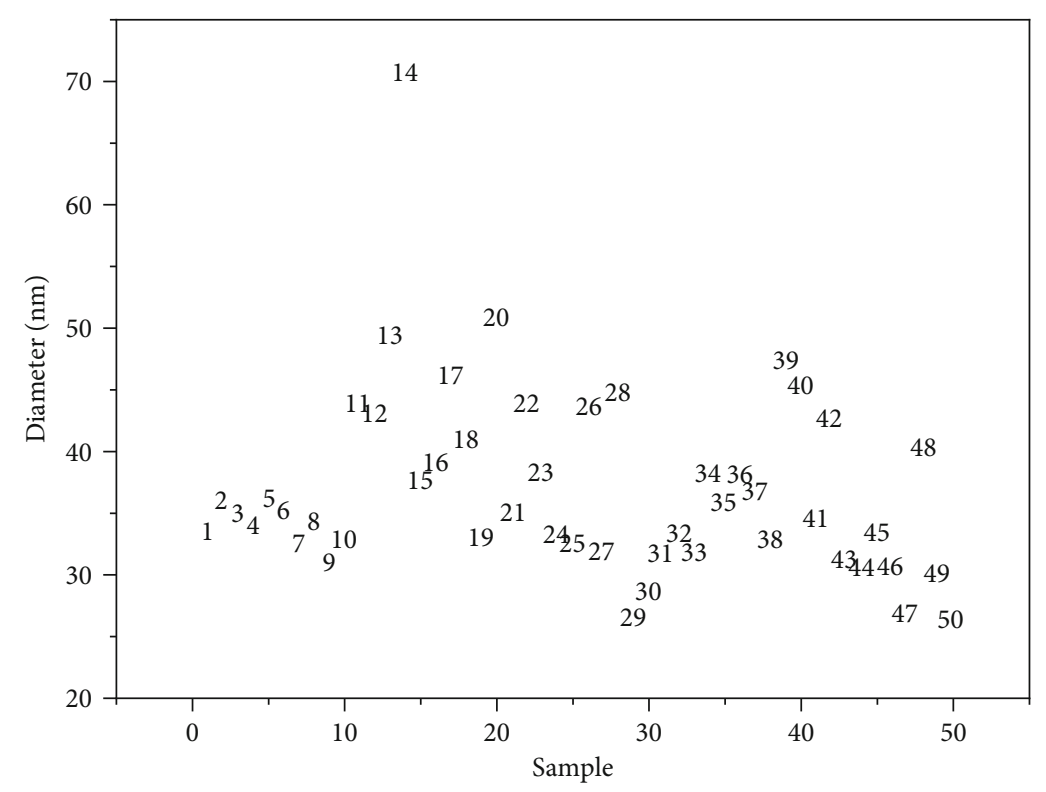

(a)

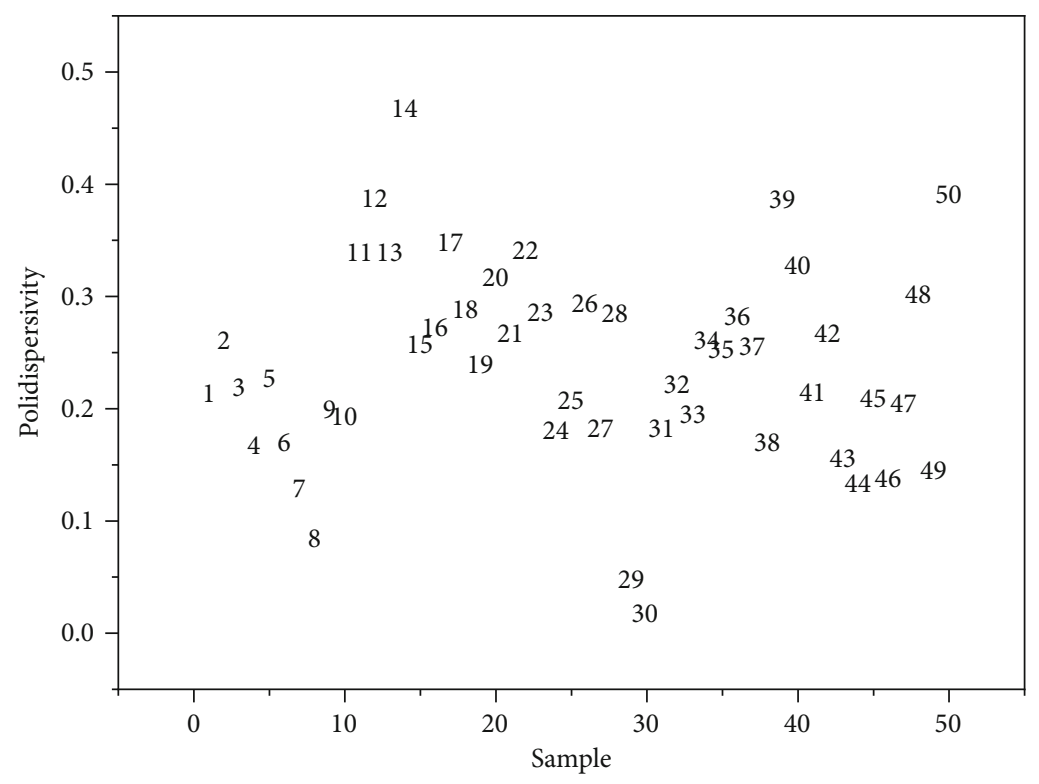

(b)

FIgURE 2: Parameter variability (a) diameter and (b) polydispersity of synthesized samples. Each sample is represented by its respective number.

techniques starts with a careful evaluation from the samples' spectra. In this stage, samples presenting spectral characteristics quite different from the others and those which do not represent extreme values of interest parameters can be excluded; working spectral range for model construction should be selected, which is often due to the exclusion of spectral regions with high noise and/or high absorbance values.

Figure 3(a) presents the spectra in the range from 200 to $700 \mathrm{~nm}$ of all synthesized samples and in Figure 3(b) the spectral range of 45 samples used during construction of multivariate calibration models. It may be noted that some samples have a spectral profile different from the others.
Also, the spectra present undesired characteristics such as noise and baseline displacement. The regions between 700-600 nm and 250-200 nm were eliminated from the construction stage of multivariate calibration models because they presented low signal-to-noise ratio and absorption of more than 1.5 a.u., respectively. In addition, the region between 550 and $600 \mathrm{~nm}$ was also excluded because, according to the literature, the region between 600 and $800 \mathrm{~nm}$ corresponds to plasmonic surface resonance frequencies (mainly in the range between 600 and $800 \mathrm{~nm}$ ) of anisotropic nanoparticles and are highly dependent on the aspect ratios and other factors [49] such as concentration of bromide ions, tricking reaction time. 


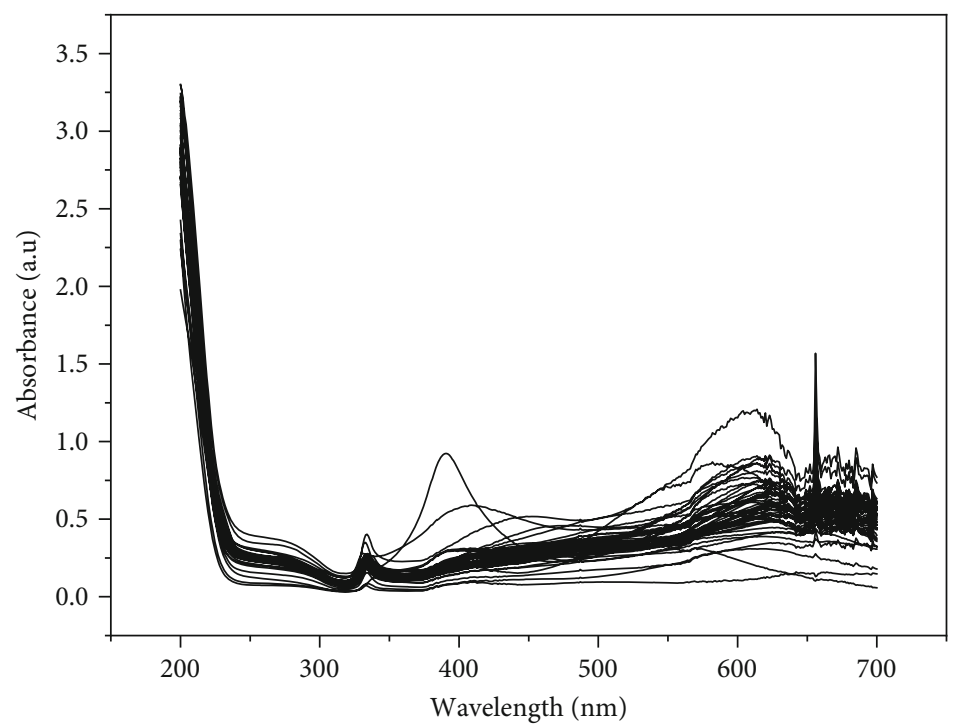

(a)

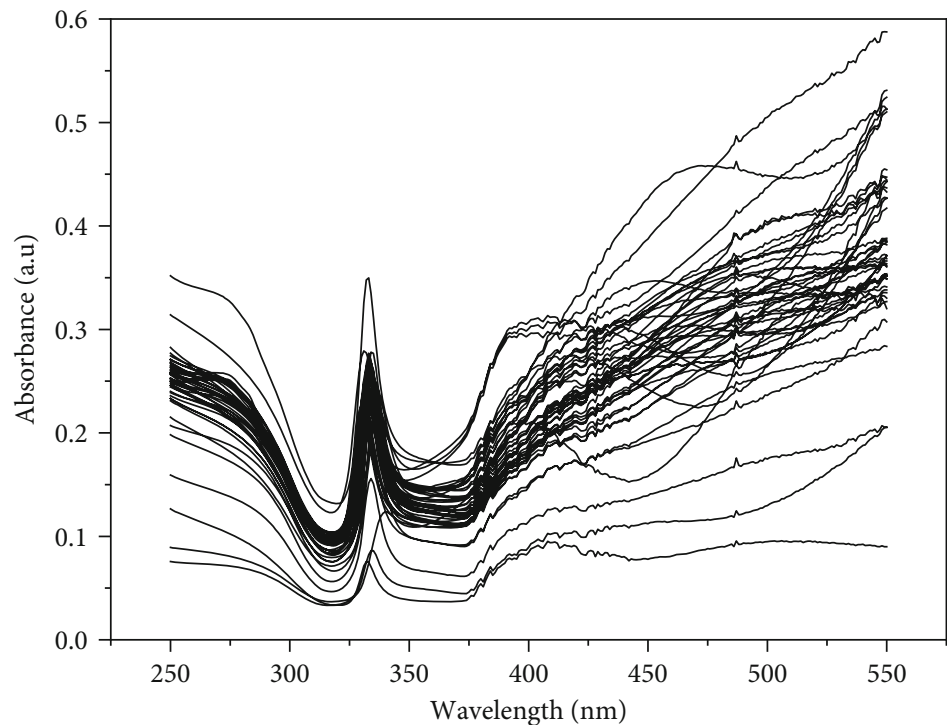

(b)

FIgURe 3: UV-Vis spectra of synthesized AgNP samples.

Hsu et al. [50] found that the SPR quadrupole band displacement in the silver nanoprism plane depends on bromide ion concentration, which binds to the nanoprism corners, favoring their truncation (Ciou et al. [51]) when its reaction time is short (less than $30 \mathrm{~min}$ ) and the aspect ratio decreasing. In another study, Brioude and Pileni [52] explain that whatever the resonance order, by increasing the aspect ratio, the positions of off-plane and plane modes evolve distinctly. Thus, for high proportions, the length of the nanodisc becomes much larger than the height, leading to an opposite behavior of mode in the plane and out of the plane and displacements in the absorption maxima. In contrast, at a low aspect ratio, out-of-plane and plane resonance modes are comparable, with near-peak absorption positions. In this work, the truncation reaction time was 3 minutes.

Furthermore, in order to expand the ranges of diameters and polydispersity, the bromide ion concentration was varied in both concentration and volume. As the bromide ion concentration is associated with aspect ratio variation, one can observe nonreproducibility in the plane of SPR quadrupole bands. The insertion of this spectral region in the construction of multivariate models resulted in models with low predictive capacity. It is also observed, from Figure 4, the displacement of a central peak in the band located between 330 and $350 \mathrm{~nm}$, referring to quadrupole resonance outside the plane. According to Brioude and Pileni [52] who used a DDA (Dipole Discrete Approximation) simulation method to determine the evolution of AgNP optical properties, these displacements may be related to imperfections, such as increased thickness or decreased edge length, rather than AgNP size, as reported in other studies [53, 54]. Thus, the selected working spectral region was 250 to $550 \mathrm{~nm}$ [55].

Any instrumental measure is composed by a signal portion related to the interest parameter and also by a noise 


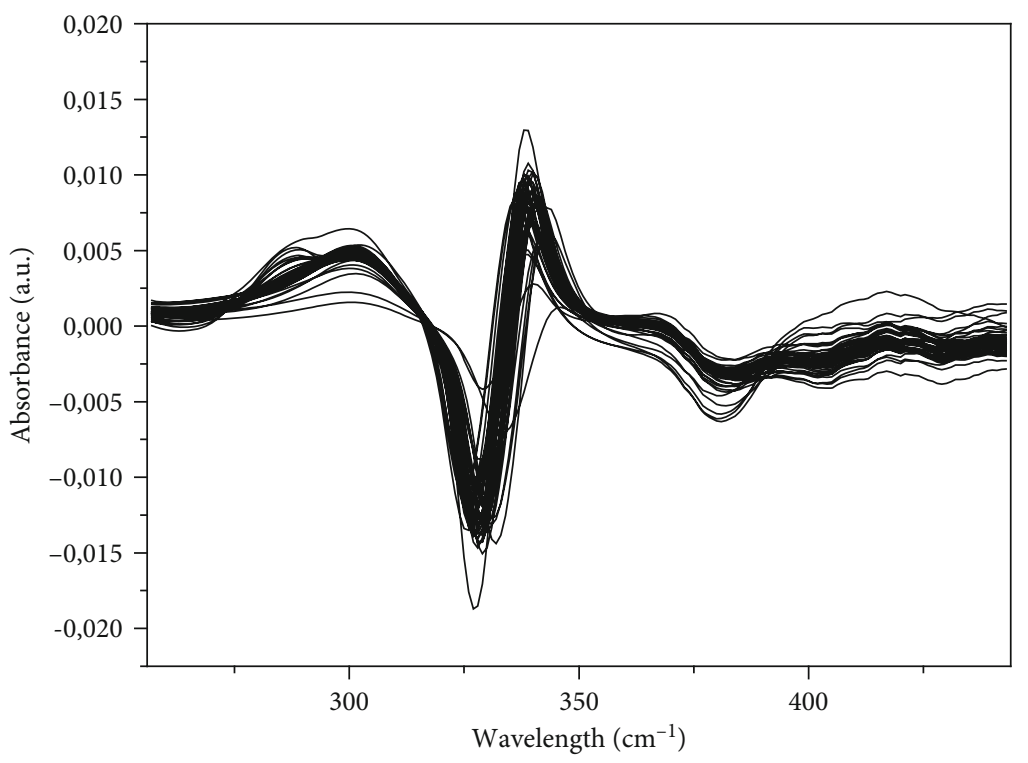

FIGURE 4: AgNp spectra with pretreatments (D1-J15-POL2).

portion which leads to calibration model imprecision. Thus, before starting the construction of multivariate models, it is necessary to submit the instrumental data to a preprocessing step in order to remove or minimize spurious information which are not related to the property being investigated. Thus, the spectral pretreatments are intended to minimize spectral noise or spectral characteristics which are not related to the interest properties, such as baseline displacement or signal scattering. In this work, MSC (Multiplicative Scatter Correction), EMSC, SNV (Standard Normal Deviation), smoothing, derivatives, and their combination were tested [56, 57].

However, prior to the application of any preprocessing to the data, it is necessary to select the samples that will be used in the calibration and prediction steps. This is a very important step in the construction of multivariate models, because in order to have an effective model, it is necessary that the used samples in the calibration step contain the variability of the problem under investigation. In this work, the selection of calibration and prediction samples was performed using a SPXY algorithm [58], which is based on both the spectral characteristics (X) and the variability of interest parameter (Y) for a more representative sample selection. There is relation between the problem complexity to be solved and the sample number to be used for the model construction (calibration stage). For systems in which small variation in the interest parameter is observed, a small calibration set is able to contemplate the latent variable relation of the interest property. In the case of samples with greater complexity, where they have a greater portion of concentration variation, the calibration set requires a larger number of samples to contemplate latent variables and interest property [59]. In this work, $2 / 3$ of samples were taken for the calibration set and $1 / 3$ for the validation set.

The study on outliers' presence based on leverage versus residue graph was performed, both in the spectral data matrix $(\mathrm{X})$ and in the matrix containing interest parameters determined by the reference methods (Y). From this tool, 4 samples with high residue and high influence were detected, both in relation to X and Y, Figures 5(a) and 5(b), respectively, which were excluded from the data set. Based on this, the calibration set was composed by 29 samples and the prediction by 15 ones.

The choice of the best pretreatment was based on values of merit figures, as root mean squared error in the steps of calibration (RMSEC), internal validation (RMSECV), and prediction (RMSEP); coefficient of determination $(R)$; correlation $\left(r^{2}\right)$; bias; slope; offset; and number of latent variables (LV) used to construct the model. The internal validation was performed by full cross-validation. The values of obtained merit figures [60] for the constructed models with raw and preprocessed data are shown in Tables 2 and 3. Only results of models with the best performance are presented.

For diameter determination, the constructed models with raw or first derivative data with a 15-point window and second-order polynomial (D1-J15-POL2) presented comparable results. For polydispersity determination, the first derivative with a 15-point window and second-order polynomial (D1-J15-POL2) was the preprocessing which presented the lowest RMSE values and lowest number of used latent variables for model construction, showing a precise and parsimonious model. Thus, in order to implement the developed methodology as routine analysis, D1-J15-POL2 was assumed as the best preprocessing for determination of two interest parameters. Figure 4 shows preprocessed UV-Vis (D1-J15POL2) spectra of AgNP samples.

In order to improve the performance of built models, a selection of spectral variables was performed based on the jackknife algorithm, which selects the most informative regression coefficients, based on Martens uncertainty test. New PLS models were developed with selected coefficients. Measured merit figure values for the new models are shown in Tables 2 and 3. 


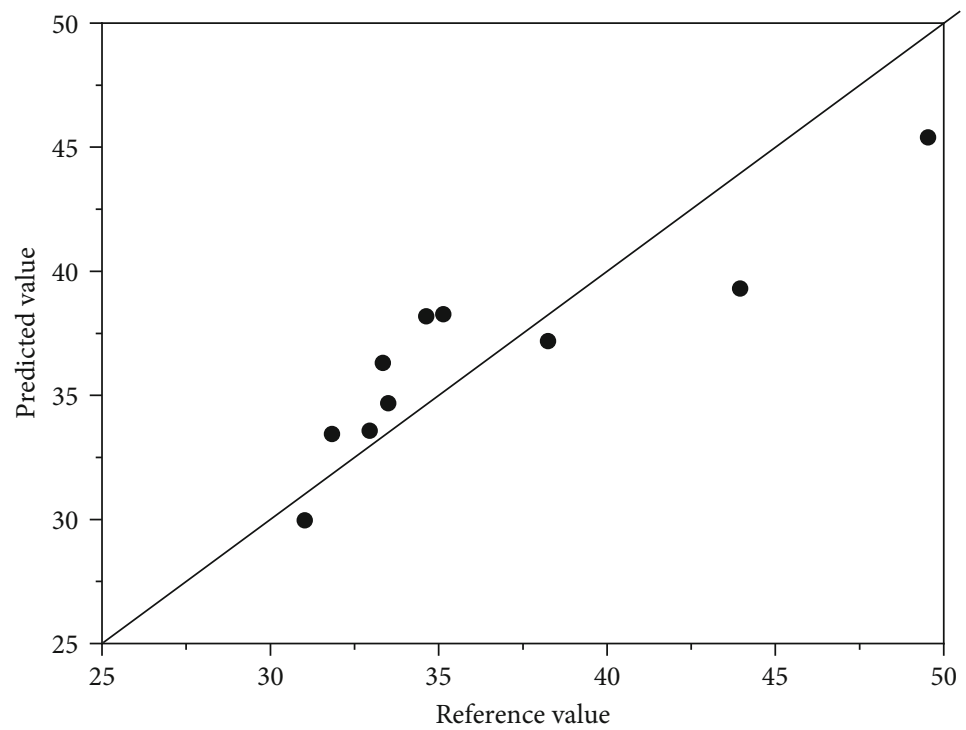

Figure 5: Plot of predicted values by the model D1-J15-POL2 versus measured values by the reference method for the diameter.

TABLE 2: Merit figures for diameter prediction-intended models.

\begin{tabular}{|c|c|c|c|c|c|c|c|c|c|}
\hline \multicolumn{10}{|c|}{ Merit figures for diameter } \\
\hline Preprocessing & Method for variable selecting & VL & Step & RMSE & Correlation & $R^{2}$ & Slope & Offset & Bias \\
\hline \multirow{2}{*}{ Raw } & \multirow{2}{*}{-} & \multirow{2}{*}{6} & $\mathrm{Cal}$ & 2.6536 & 0.8635 & 0.7456 & 0.7456 & 9.27196 & 0 \\
\hline & & & Pred. & 5.6666 & 0.5945 & 0.2939 & 0.2281 & 28.0886 & -0.8274 \\
\hline \multirow{2}{*}{ Raw } & \multirow{2}{*}{$\mathrm{JK}$} & \multirow{2}{*}{4} & Cal & 3.0167 & 0.8123 & 0.6599 & 0.6599 & 12.396 & 0 \\
\hline & & & Pred. & 6.1412 & 0.4381 & 0.1707 & 0.1544 & 30.8809 & -0.7946 \\
\hline \multirow{2}{*}{ D1-J15-POL2 } & \multirow{2}{*}{-} & \multirow{2}{*}{5} & Cal & 2.2741 & 0.9057 & 0.8204 & 0.8204 & 6.5586 & 0 \\
\hline & & & Pred. & 2.9779 & 0.8369 & 0.7136 & 0.7772 & 8.1825 & 0.0461 \\
\hline \multirow{2}{*}{ D1-J15-POL2 } & \multirow{2}{*}{ JK } & \multirow{2}{*}{6} & $\mathrm{Cal}$ & 2.1209 & 0.9185 & 0.8437 & 0.8437 & 5.7045 & 0 \\
\hline & & & Pred. & 2.7658 & 0.8916 & 0.7586 & 0.6282 & 13.745 & 0.2062 \\
\hline
\end{tabular}

VL: latent or spectral variables; Cal: calibration step; Pred.: prediction stage; JK: model built with jackknife coefficients; D1-J15-POL2: 1st derivative window of 15-point polynomial of 2nd order; D1-J15-POL2 (JK): constructed models with selected samples prior to preprocessing application.

TABLE 3: Merit figures for polydispersity prediction.

\begin{tabular}{|c|c|c|c|c|c|c|c|c|c|}
\hline \multicolumn{10}{|c|}{ Merit figures for polydispersity } \\
\hline Preprocessing & Method for variable selecting & VL & Step & RMSE & Correlation & $R^{2}$ & Slope & Offset & Bias \\
\hline \multirow{2}{*}{ Raw } & \multirow{2}{*}{ - } & \multirow{2}{*}{6} & $\mathrm{Cal}$ & 0.04253 & 0.9037 & 0.8166 & 0.8166 & 0.0440 & 0 \\
\hline & & & Pred. & 0.04037 & 0.8123 & 0.5766 & 0.8657 & 0.0238 & -0.0085 \\
\hline \multirow{2}{*}{ Raw } & \multirow{2}{*}{$\mathrm{JK}$} & \multirow{2}{*}{1} & $\mathrm{Cal}$ & 0.07827 & 0.6264 & 0.3923 & 0.3923 & 0.1472 & 0 \\
\hline & & & Pred. & 0.05624 & 0.4721 & 0.1782 & 0.1248 & 0.2088 & -0.0024 \\
\hline \multirow{2}{*}{ D1-J15-POL2 } & \multirow{2}{*}{ - } & \multirow{2}{*}{6} & $\mathrm{Cal}$ & 0.03995 & 0.9226 & 0.8512 & 0.8512 & 0.0358 & 0 \\
\hline & & & Pred. & 0.05728 & 0.8399 & 0.7141 & 0.7937 & 0.0514 & 0.0018 \\
\hline \multirow{2}{*}{ D1-J15-POL2 } & \multirow{2}{*}{ JK } & \multirow{2}{*}{6} & Cal & 0.04110 & 0.9178 & 0.8425 & 0.8425 & 0.0378 & 0 \\
\hline & & & Pred. & 0.02076 & 0.9295 & 0.8299 & 0.9945 & -0.004 & -0.0060 \\
\hline
\end{tabular}

VL: latent or spectral variables; Cal: calibration step; Pred.: prediction stage; JK: model built with jackknife coefficients; D1-J15-POL2: 1st derivative window of 15-point polynomial of 2nd order; D1-J15-POL2 (JK): constructed models with selected samples prior to preprocessing application. 


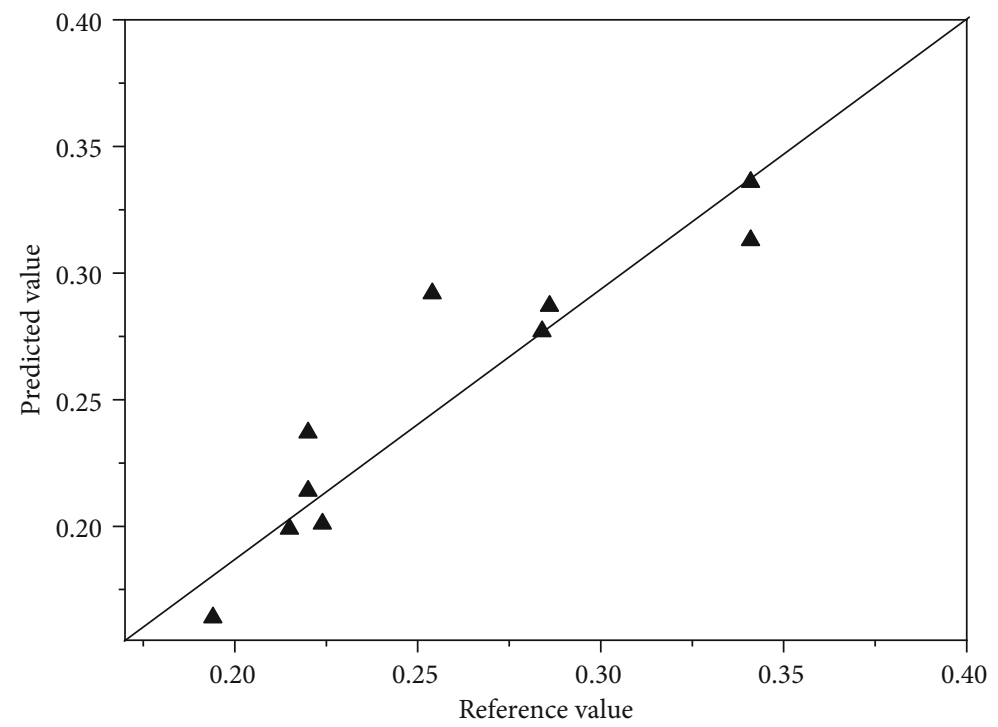

FIgURe 6: Plot of predicted values by the model D1-J15-POL2-JK versus measured values by the reference method for polydispersity.

Analyzing the merit figures as RMSE and $R^{2}$, variable numbers which describe predictive performance, it was observed that the model D1-J15-POL2-JK is the most suitable for parameters' prediction of diameter and polydispersity. The D1-J15-POL2 model, constructed with all spectral variables, and the D1-J15-POL2-JK model, constructed with the most informative regression coefficients selected by the jackknife, presented comparable RMSEC, RMSECV, and RMSEP values; however, the first one used a smaller number of VL. In addition to the models' practical application, the fewer the steps applied during calibration, the easier it will be used. Based on these arguments the D1-J15-POL2 model was chosen in the prediction step.

Figures 5 and 6 show plots of predicted values by the model versus measured values by the reference method for diameter and polydispersity, respectively, which demonstrate agreement between predicted and measured values. One can observe the random distribution of the samples around the bisector, which shows there are no trends in the results.

\section{Conclusions}

Reference methodologies used for AgNP characterization, although precise, are laborious techniques, and most of them have higher cost. On the other hand, UV-Vis spectroscopy combined with chemometrics appears as a reasonable alternative to evaluate parameters such as diameter and polydispersity, since after the model development, the use of conventional technics are less required in routine analyses. The constructed models with the entire spectral range presented better performance than those built with the jackknife coefficients, since they used a lower number of latent variables. The measured error for diameter prediction was $5.31 \%$, and for polydispersity, it was $4.43 \%$, being acceptable for the purposes of the present work. Summing up, this work has successfully developed a proper model, which provides savings of time and costs to acquire information related to size, polydispersity, morphology, and spectral characteristics of silver nanoparticles through the UV-Vis technique associated with a chemometric model, and the methods and data used are described here.

\section{Data Availability}

The developed chemiometric model as well as raw data used to support the findings of this study will be available then requested from the corresponding author.

\section{Conflicts of Interest}

The authors declare that there is no conflict of interest regarding the publication of this paper.

\section{Acknowledgments}

The authors are grateful to CAPES, to the Research Group on Chemometrics and Advanced Materials for Sustainability of the Semiarid-QUIMASSA represented by Professor Simone Simões and to the Northeastern Biomaterials Evaluation and Development Laboratory (CERTBIO) and the Federal University of Campina Grande (UFCG). This work was supported by the Higher Education Personnel Improvement Coordination-CAPES. Prof Marcus Fook and Prof Renate Wellen are $\mathrm{CNPq}$ fellows.

\section{References}

[1] A. Baranwal, A. K. Chiranjivi, A. Kumar, V. K. Dubey, and P. Chandra, "Design of commercially comparable nanotherapeutic agent against human disease-causing parasite, Leishmania," Scientific Reports, vol. 8, no. 1, p. 8814, 2018.

[2] B. Han, Y. L. Zhang, L. Zhu et al., "Direct laser scribing of AgNPs@RGO biochip as a reusable SERS sensor for DNA detection," Sensors and Actuators B: Chemical, vol. 270, pp. 500-507, 2018. 
[3] B. Shao, X. Ma, S. Zhao et al., "Nanogapped Au(core) @ Au-Ag(shell) structures coupled with $\mathrm{Fe} 3 \mathrm{O} 4$ magnetic nanoparticles for the detection of Ochratoxin A," Analytica Chimica Acta, vol. 1033, pp. 165-172, 2018.

[4] J. Pulit-Prociak, J. Chwastowski, M. Siudek, and M. Banach, "Incorporation of metallic nanoparticles into cosmetic preparations and assessment of their physicochemical and utility properties," Journal of Surfactants and Detergents, vol. 21, no. 4, pp. 575-591, 2018.

[5] X. Xuan, J. Y. Kim, X. Hui, P. S. Das, H. S. Yoon, and J.-Y. Park, "A highly stretchable and conductive $3 \mathrm{D}$ porous graphene metal nanocomposite based electrochemical-physiological hybrid biosensor," Biosensors and Bioelectronics, vol. 120, pp. 160-167, 2018.

[6] Y. Mou, Y. Zhang, H. Cheng, Y. Peng, and M. Chen, "Fabrication of highly conductive and flexible printed electronics by low temperature sintering reactive silver ink," Applied Surface Science, vol. 459, pp. 249-256, 2018.

[7] E. Ahmadian, S. M. Dizaj, E. Rahimpour et al., "Effect of silver nanoparticles in the induction of apoptosis on human hepatocellular carcinoma (HepG2) cell line," Materials Science and Engineering: C, vol. 93, pp. 465-471, 2018.

[8] F. M. Zahed, B. Hatamluyi, F. Lorestani, and Z. Es'haghi, "Silver nanoparticles decorated polyaniline nanocomposite based electrochemical sensor for the determination of anticancer drug 5-fluorouracil," Journal of Pharmaceutical and Biomedical Analysis, vol. 161, pp. 12-19, 2018.

[9] C. Tonda-Turo, F. Ruini, C. Ceresa et al., "Nanostructured scaffold with biomimetic and antibacterial properties for wound healing produced by 'green electrospinning,", Colloids and Surfaces B: Biointerfaces, vol. 172, pp. 233-243, 2018.

[10] D. Kharaghani, Y. Kee Jo, M. Q. Khan, Y. Jeong, H. J. Cha, and I. S. Kim, "Electrospun antibacterial polyacrylonitrile nanofiber membranes functionalized with silver nanoparticles by a facile wetting method," European Polymer Journal, vol. 108, pp. 69-75, 2018.

[11] K. Nešović, A. Janković, V. Kojić et al., "Silver/poly(vinyl alcohol)/chitosan/graphene hydrogels - Synthesis, biological and physicochemical properties and silver release kinetics," Composites Part B: Engineering, vol. 154, pp. 175-185, 2018.

[12] C. E. Escárcega-González, J. A. Garza-Cervantes, A. VazquezRodríguez et al., "In vivo antimicrobial activity of silver nanoparticles produced via a green chemistry synthesis using Acacia rigidula as a reducing and capping agent," International Journal of Nanomedicine, vol. Volume 13, pp. 2349-2363, 2018.

[13] G. Moreno-Martin, M. E. León-Gonzalez, and Y. Madrid, "Simultaneous determination of the size and concentration of AgNPs in water samples by UV-vis spectrophotometry and chemometrics tools," Talanta, vol. 188, pp. 393-403, 2018.

[14] A.-L. Kubo, I. Capjak, I. V. Vrček et al., "Antimicrobial potency of differently coated 10 and $50 \mathrm{~nm}$ silver nanoparticles against clinically relevant bacteria Escherichia coli and Staphylococcus aureus," Colloids and Surfaces B: Biointerfaces, vol. 170, pp. 401-410, 2018.

[15] H. Singh, J. Du, P. Singh, and T. H. Yi, "Extracellular synthesis of silver nanoparticles by Pseudomonas sp. THG-LS1.4 and their antimicrobial application," Journal of Pharmaceutical Analysis, vol. 8, no. 4, pp. 258-264, 2018.

[16] D. J. Gorth, D. M. Rand, and T. J. Webster, "Silver nanoparticle toxicity in Drosophila: size does matter," International Journal of Nanomedicine, vol. 6, p. 343, 2011.
[17] K. Mehennaoui, S. Cambier, T. Serchi et al., "Do the pristine physico-chemical properties of silver and gold nanoparticles influence uptake and molecular effects on Gammarus fossarum (Crustacea Amphipoda)?," Science of the Total Environment, vol. 643, pp. 1200-1215, 2018.

[18] N. T.-P. Nguyen, L. V. H. Nguyen, N. T. Thanh et al., "Stabilization of silver nanoparticles in chitosan and gelatin hydrogel and its applications," Materials Letters, vol. 248, pp. 241-245, 2019.

[19] M. D. Scherer, J. C. V. Sposito, W. F. Falco et al., "Cytotoxic and genotoxic effects of silver nanoparticles on meristematic cells of Allium cepa roots: a close analysis of particle size dependence," Science of the Total Environment, vol. 660, pp. 459-467, 2019.

[20] P. Banerjee, M. Satapathy, A. Mukhopahayay, and P. Das, "Leaf extract mediated green synthesis of silver nanoparticles from widely available Indian plants: synthesis, characterization, antimicrobial property and toxicity analysis," Bioresources and Bioprocessing, vol. 1, no. 1, p. 3, 2014.

[21] A. Bootz, V. Vogel, D. Schubert, and J. Kreuter, "Comparison of scanning electron microscopy, dynamic light scattering and analytical ultracentrifugation for the sizing of poly (butyl cyanoacrylate) nanoparticles," European Journal of Pharmaceutics and Biopharmaceutics, vol. 57, no. 2, pp. 369-375, 2004.

[22] A. Michna, M. Morga, Z. Adamczyk, and K. Kubiak, "Monolayers of silver nanoparticles obtained by green synthesis on macrocation modified substrates," Materials Chemistry and Physics, vol. 227, pp. 224-235, 2019.

[23] K. L. Planken, B. W. Kuipers, and A. P. Philipse, "Model independent determination of colloidal silica size distributions via analytical ultracentrifugation," Analytical Chemistry, vol. 80, no. 23, pp. 8871-8879, 2008.

[24] D. Mahl, J. Diendorf, W. Meyer-Zaika, and M. Epple, "Possibilities and limitations of different analytical methods for the size determination of a bimodal dispersion of metallic nanoparticles," Colloids and Surfaces A: Physicochemical and Engineering Aspects, vol. 377, no. 1-3, pp. 386-392, 2011.

[25] A. R. Poda, A. J. Bednar, A. J. Kennedy et al., "Characterization of silver nanoparticles using flow-field flow fractionation interfaced to inductively coupled plasma mass spectrometry," Journal of Chromatography A, vol. 1218, no. 27, pp. 42194225, 2011.

[26] K. Kalimuthu, R. Suresh Babu, D. Venkataraman, M. Bilal, and S. Gurunathan, "Biosynthesis of silver nanocrystals by Bacillus licheniformis," Colloids and Surfaces B: Biointerfaces, vol. 65, no. 1, pp. 150-153, 2008.

[27] J. Du, Z. Hu, W.-j. Dong, Y. Wang, S. Wu, and Y. Bai, "Biosynthesis of large-sized silver nanoparticles using Angelica keiskei extract and its antibacterial activity and mechanisms investigation," Microchemical Journal, vol. 147, pp. 333-338, 2019.

[28] W. Haiss, N. T. K. Thanh, J. Aveyard, and D. G. Fernig, "Determination of size and concentration of gold nanoparticles from UV-Vis spectra," Analytical Chemistry, vol. 79, no. 11, pp. 4215-4221, 2007.

[29] E. Tomaszewska, K. Soliwoda, K. Kadziola et al., "Detection limits of DLS and UV-Vis spectroscopy in characterization of polydisperse nanoparticles colloids," Journal of Nanomaterials, vol. 2013, Article ID 313081, 10 pages, 2013.

[30] N. L. Pacioni, C. D. Borsarelli, V. Rey, and A. V. Veglia, "Synthetic routes for the preparation of silver nanoparticles," in Silver nanoparticle applications, pp. 13-46, Springer, 2015. 
[31] B. N. Khlebtsov and N. G. Khlebtsov, "On the measurement of gold nanoparticle sizes by the dynamic light scattering method," Colloid Journal, vol. 73, no. 1, pp. 118-127, 2011.

[32] M. Zimbone, L. Calcagno, G. Messina, P. Baeri, and G. Compagnini, "Dynamic light scattering and UV-vis spectroscopy of gold nanoparticles solution," Materials Letters, vol. 65, no. 19-20, pp. 2906-2909, 2011.

[33] B. J. Berne and R. Pecora, Dynamic Light Scattering: with Applications to Chemistry, Biology, and Physics, Courier Corporation, 2000.

[34] D. Paramelle, A. Sadovoy, S. Gorelik, P. Free, J. Hobley, and D. G. Fernig, "A rapid method to estimate the concentration of citrate capped silver nanoparticles from UV-visible light spectra," Analyst, vol. 139, no. 19, pp. 4855-4861, 2014.

[35] C. Pasquini, "Near infrared spectroscopy: a mature analytical technique with new perspectives-a review," Analytica Chimica Acta, vol. 1026, pp. 8-36, 2018.

[36] N. Kumar, A. Bansal, G. Sarma, and R. K. Rawal, "Chemometrics tools used in analytical chemistry: an overview," Talanta, vol. 123, pp. 186-199, 2014.

[37] W. L. Hinze, H. N. Singh, Y. Baba, and N. G. Harvey, "Micellar enhanced analytical fluorimetry," TrAC Trends in Analytical Chemistry, vol. 3, no. 8, pp. 193-199, 1984.

[38] R. Bro, "Multivariate calibration: what is in chemometrics for the analytical chemist?," Analytica Chimica Acta, vol. 500, no. 1-2, pp. 185-194, 2003.

[39] F. Rabbani, M. R. Hormozi Nezhad, and H. Abdollahi, "Useful multivariate kinetic analysis: Size determination based on cystein- induced aggregation of gold nanoparticles," Spectrochimica Acta Part A: Molecular and Biomolecular Spectroscopy, vol. 115, pp. 588-594, 2013.

[40] C. Jing and Y. Fang, "Experimental (SERS) and theoretical (DFT) studies on the adsorption behaviors of L-cysteine on gold/silver nanoparticles," Chemical Physics, vol. 332, no. 1, pp. 27-32, 2007.

[41] Q. Zhang, N. Li, J. Goebl, Z. Lu, and Y. Yin, "A systematic study of the synthesis of silver nanoplates: is citrate a "magic reagent?"," Journal of the American Chemical Society, vol. 133, no. 46, pp. 18931-18939, 2011.

[42] G. Panzarasa, "Just what is it that makes silver nanoprisms so different, so appealing?," Journal of Chemical Education, vol. 92, no. 11, pp. 1918-1923, 2015.

[43] G. Mie, "Articles on the optical characteristics of turbid tubes, especially colloidal metal solutions," Annalen der Physik, vol. 25, no. 3, pp. 377-445, 1908.

[44] R. Jin, Y. Cao, C. A. Mirkin, K. Kelly, G. C. Schatz, and J. Zheng, "Photoinduced conversion of silver nanospheres to nanoprisms," Science, vol. 294, no. 5548, pp. 1901-1903, 2001.

[45] S. Chen and D. L. Carroll, "Synthesis and characterization of truncated triangular silver nanoplates," Nano Letters, vol. 2, no. 9, pp. 1003-1007, 2002.

[46] J. Chalmers and P. Griffiths, Handbook of Vibrational Spectroscopy, 5 Volumes Set, Wiley, 2002.

[47] S. Farooq, F. D. Nunes, and R. E. de Araujo, "Optical properties of silver nanoplates and perspectives for biomedical applications," Photonics and Nanostructures-Fundamentals and Applications, vol. 31, pp. 160-167, 2018.

[48] M. Cloutier, S. Turgeon, Y. Busby, M. Tatoulian, J.-J. Pireaux, and D. Mantovani, "Controlled distribution and clustering of silver in Ag-DLC nanocomposite coatings using a hybrid plasma approach," ACS Applied Materials \& Interfaces, vol. 8, no. 32, pp. 21020-21027, 2016.

[49] K. L. Kelly, E. Coronado, L. L. Zhao, and G. C. Schatz, The optical properties of metal nanoparticles: the influence of size, shape, and dielectric environment, ACS Publications, 2003.

[50] M. S. Hsu, Y. W. Cao, H. W. Wang, Y. S. Pan, B. H. Lee, and C. L. Huang, "Time-dependent surface plasmon resonance spectroscopy of silver nanoprisms in the presence of halide ions," ChemPhysChem, vol. 11, no. 8, pp. 1742-1748, 2010.

[51] S.-H. Ciou, Y.-W. Cao, H.-C. Huang, D.-Y. Su, and C.-L. Huang, "SERS enhancement factors studies of silver nanoprism and spherical nanoparticle colloids in the presence of bromide ions," The Journal of Physical Chemistry C, vol. 113, no. 22, pp. 9520-9525, 2009.

[52] A. Brioude and M. Pileni, "Silver nanodisks: optical properties study using the discrete dipole approximation method," The Journal of Physical Chemistry B, vol. 109, no. 49, pp. 2337123377, 2005.

[53] Q. Zhang, Y. Hu, S. Guo, J. Goebl, and Y. Yin, "Seeded growth of uniform Ag nanoplates with high aspect ratio and widely tunable surface plasmon bands," Nano Letters, vol. 10, no. 12, pp. 5037-5042, 2010.

[54] N. Li, Q. Zhang, S. Quinlivan, J. Goebl, Y. Gan, and Y. Yin, " $\mathrm{H}_{2} \mathrm{O}_{2}$-aided seed-mediated synthesis of silver nanoplates with improved yield and efficiency," ChemPhysChem, vol. 13, no. 10, pp. 2526-2530, 2012.

[55] D. P. M. Ballottin, Caracterização de nanopartículas de prata e sua aplicação na produção de tecidos antimicrobianos, 2014.

[56] J. Skogholt, K. H. Liland, and U. G. Indahl, "Preprocessing of spectral data in the extended multiplicative signal correction framework using multiple reference spectra," Journal of Raman Spectroscopy, vol. 50, no. 3, pp. 407-417, 2018.

[57] M. J. Martelo-Vidal, F. Domínguez-Agis, and M. Vázquez, "Ultraviolet/visible/near-infrared spectral analysis and chemometric tools for the discrimination of wines between subzones inside a controlled designation of origin: a case study of Rías Baixas," Australian Journal of Grape and Wine Research, vol. 19, no. 1, pp. 62-67, 2013.

[58] R. Galvao, M. Araujo, G. Jose, M. Pontes, E. Silva, and T. Saldanha, "A method for calibration and validation subset partitioning," Talanta, vol. 67, no. 4, pp. 736-740, 2005.

[59] P. Q. Guimarães, Metodologia baseada em NIRS e quimiometria para a determinação de parâmetros de qualidade da quitosana para fins biomédicos, 2017.

[60] A. C. Olivieri, H. C. Goicoechea, and F. A. Inón, "MVC1: an integrated MatLab toolbox for first-order multivariate calibration," Chemometrics and Intelligent Laboratory Systems, vol. 73, no. 2, pp. 189-197, 2004. 